\author{
Military Technical College \\ Kobry El-Kobbah, \\ Cairo, Egypt.
}

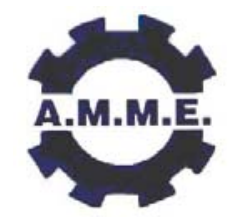

\title{
COMBINED EFFECT OF INCIDENCE - FOREBODY ANGLES ON THE FLOW STRUCTURE INSIDE SPIKED SUPERSONIC INTAKES
}

\author{
AL-KAYIEM* H.H. and SALIH** T.W.
}

\begin{abstract}
A high static pressure at the inlet face and minimal total pressure loss at the intake entry are required for an efficient operation of the spiked supersonic jet engines. The turning (Forebody) angles are optimized for a maximum total pressure recovery at the intake entry for the design Mach number condition, so that the compression shocks fall on the cowl lip. Non zero incidence angle changes the effective deflection angle up and down the spike, so the combining effect of incidence and forebody angles is essential for the intake performance. The present analysis investigates the pressure distribution at the compressor face at different forebody-Incident combinations at different supersonic fly speeds ranging from 1.8 to 2.2 Mach. The numerical analysis is carried out under 2-D, steady and viscous flow assumptions. The external flow, which consists of the set of waves, is solved analytically. CFD analysis using Control Volume Formulation technique is applied to analyze the internal flow. In house program is built to solve the governing sets of equations by using SIMPLE algorithm. The analyses are carried out at incidence angles ranging from $0^{\circ}$ to $10^{\circ}$ and forebody angles ranging from $6^{\circ}$ to $25^{\circ}$. The procedure has been verified by comparing with previous experimental results. The results obtained have generally shown, that the pressure recovery increases by the increasing of the wedge angle and decreases by increasing of incidence angle. In some operating conditions, the combination of the three factors, Mach no., the incidence, and the forebody angle results in a complicated mechanism due to the presence of compression and expansion waves in the external part of the intake simultaneously.
\end{abstract}

\section{KEYWORDS}

Supersonic flow, Inlets, CFD, Spike intakes, Pressure recovery.

\footnotetext{
* Associate Professor, Mech. Eng. Dept., Universiti Teknologi PETRONAS, Bandar Seri Iskandar, 31750 Tronoh, Perak,Malaysia, Email: Hussain kayiem@petronas.com.my

** Assistant Lecturer, Mech. Eng. Dept. Al-Mustansiriyah University, Baghdad, Iraq, Tel.: +964 770254 1260, Email:tawfeeq1978@yahoo.com
} 


\section{INTRODUCTION}

The aircraft Aero-dynamists are interested in obtaining details of the flow around the outsides of the intakes. Aero-engine designers, on the other hand, are interested in details of the flow structure inside the intakes. The supersonic engines are provided with inlet features which can maintain various pre-designed operation conditions [1]. The type of intake without spike is quite unsuitable for supersonic flight conditions. There would be a normal shock wave at the mouth which causes a very sharp pressure rise with big velocity reduction. To achieve smooth functional operation of the supersonic engines, a spike is installed in the intake to provide shock series outside the intake frontal face. The spike may be fixed or may be movable. The modern jet engines are designed with movable spikes to provide variable geometry intakes [2]. Such types of spikes are required mainly for two objectives. Firstly, to produce a variable throat area that is large enough, to maintain the design air mass flow rate. Secondly, to control the shock wave system created in the external portion of the intake. Many factors are affecting the performance of the supersonic intakes. Some factors are operational factors and some are design factors. The majors among the operational factors are the angle of attack (AOA), $\alpha$, and the fly Mach no. On the other hand, the main design factor is the spike deflection angle, $\delta$ or usually called the forebody angle. The spikes may have one (single) deflection feature, two deflections feature or may be designed with curvature surface to produce smooth expansion waves. In the present study, the single deflection, 2-D spike is subjected to analytical and computational analysis.

\section{Experimental Backgrounds on Supersonic Intakes}

In contrast to many mechanical engineering fields, detailed information and clear discussion of the subject of supersonic spiked intakes are not widely available. The first serious work on supersonic inlets was done by Oswatitsch [3]* in Germany during the World War II when he performed a series of pioneering analytical and experimental investigation on a single and double wedge intake. Then after, the American started a series of investigations on supersonic intakes with conical center bodies. Englert and Obery [4] carried out investigation of 16 inch ram-jet engine in NACA Lewis supersonic wind tunnel at 1.7, 1.9 and 2 free Mach No. at $0^{\circ}$ to $10^{\circ}$ angles of attack. An extension to the work was made by Gorton [5] to study the practicability of a translating spike intake. The second series of investigations carried out by NACA was applied on double deflection conical spikes. Jones et al [6] have conducted experiments on conical spike with 22 and 35 half angles and the tests indicated that the total pressure recovery gradually decreases by increase of the free stream Mach No. Connor and Meyer [7] conducted another experimental program in NACA Lewis Laboratory on conical spikes with 20 and 28 half angles. They claimed 0.92 total pressure recovery at zero AOA. In summer 1959, Lockheed Skunk Works designed the SR-71 supersonic engine which operates up to 2 Mach. The SR-71 engine intake used variable inlet geometry by movable axisymmetric spike. The spike was found to provide higher pressure recovery at the compressor face [2].

\section{Numerical Background on Supersonic Intakes}

In the mid of eighties, a trend towards the numerical techniques has been adopted to

\footnotetext{
* Extracted from Reference 3
} 
reduce time and cost of supersonic intakes analysis, compared to the experimental investigations. Biringer [8] presented numerical solution of Euler equations based on implicit, time- marching, finite difference technique to analyze the subsonic and supersonic regions in the intake. The solution is found to converge rapidly for supercritical interpolation, while it converges slowly for the sub-critical intake operation. Moretti [9] presented an effective 2-D Euler computational technique at any number of shocks of any shape any type. Dudek [10] solved numerically for the turbulent, subsonic flow in pipe attached to conical diffusing section with $5^{\circ}$ diffusing angle. The computations were performed to set a number of iterations until the convergence histories were leveled out. Abbood [11], carried out numerical time dependent analysis of 2-D convergent-divergent nozzle. The procedure is based on the explicit; second order accurate McCormack predictor corrector scheme. This finite difference method required an axis transformation from the physical to computational domain. Following the experimental work of Yanta et al. [12] on the 2-D scramjet inlet, Gokhale and Venkat [13] have carried out numerical analysis adopting the same geometry of the inlet of $10^{\circ}$ single deflection wedge with only zero angle of attack at 2.5 Mach and 3 Mach. They have assumed viscous adiabatic flow. Their results are in good agreement with the experimental data and the analysis is capable to capture the shock waves and their interaction with boundary layer. Fayadh [14] carried out the solution of the external supersonic flow of the intake using McCormack explicit method for solving viscous, 2-D, full Navier - Stokes equations. The results show that great deal of computation time is needed to solve these problems with explicit methods. Al-Kayiem and Sharief [15] studied the case of single deflection spike of non-viscous flow in 2-D supersonic intake. The analysis has subdivided the problem into two parts. The external part is solved analytically and the properties of the flow across the shock wave have been determined. The internal part which represents the diffuser flow has been solved numerically by the solution of Euler equation using Newton-Raphson iterative method with assistance of axis transformations from physical to computational technique. The analysis was carried out for different AOA ranging from $0^{\circ}$ to $20^{\circ}$ at free stream Mach ranging from 1.4 to 3 .

Aerospace firms using their own financial resources have developed computer codes, which are not available for general use, that do a reasonable job of supersonic intake design and prediction of its performance. A 2-D computer code called (CATHI) developed by McDonnell Douglas is based on oblique shock theory. It is applicable to all 2-D inlets with single wedge and multiple wedges. (AXISPX) an axisymmetric computer code, is also developed for isentropic spike compression and employs the method of characteristics for flow field prediction and does not have angle of attack capability. (NPARC) a CFD code for aerodynamics is a partnership between the NACA Glenn Research Center (GRC) and the Arnold Engineering Development Center (AEDC). It contains of large applications and supplies a lot of examples, but does not consist of applications which combine the supersonic flow filed analysis in the intake at various angles of attack and different forebody angles.

The present study is aiming to solve and analyze the flow in supersonic intakes with single deflected wedge.

The objective is to solve and analyze the flow in supersonic intakes of single deflected wedge at various none zero AOA and Mach numbers (operational conditions) and different wedge angles (design conditions). It can be summarized as: (i) Characterizing the waves system and (ii) to predict and analyze the flow field inside the diffuser 
resulting from the various combinations of operational conditions with the design conditions.

The solution methodology adopted in the present analysis is divided the spike into two flow regimes. The external flow is solved by analytical modeling using the wave's relations, while, the internal flow is predicted numerically by solving Navier-Stokes equations under the assumptions of 2-D, viscous, steady, compressible flow. The governing equations have been solved numerically by discretizing the flow field by "Control Volume Formulation, CVF". The preliminary assumption of the flow variables at each point is obtained by applying the 1-D formulas of the viscous, compressible flow. The methodology is summarized in the flow chart, Fig.1

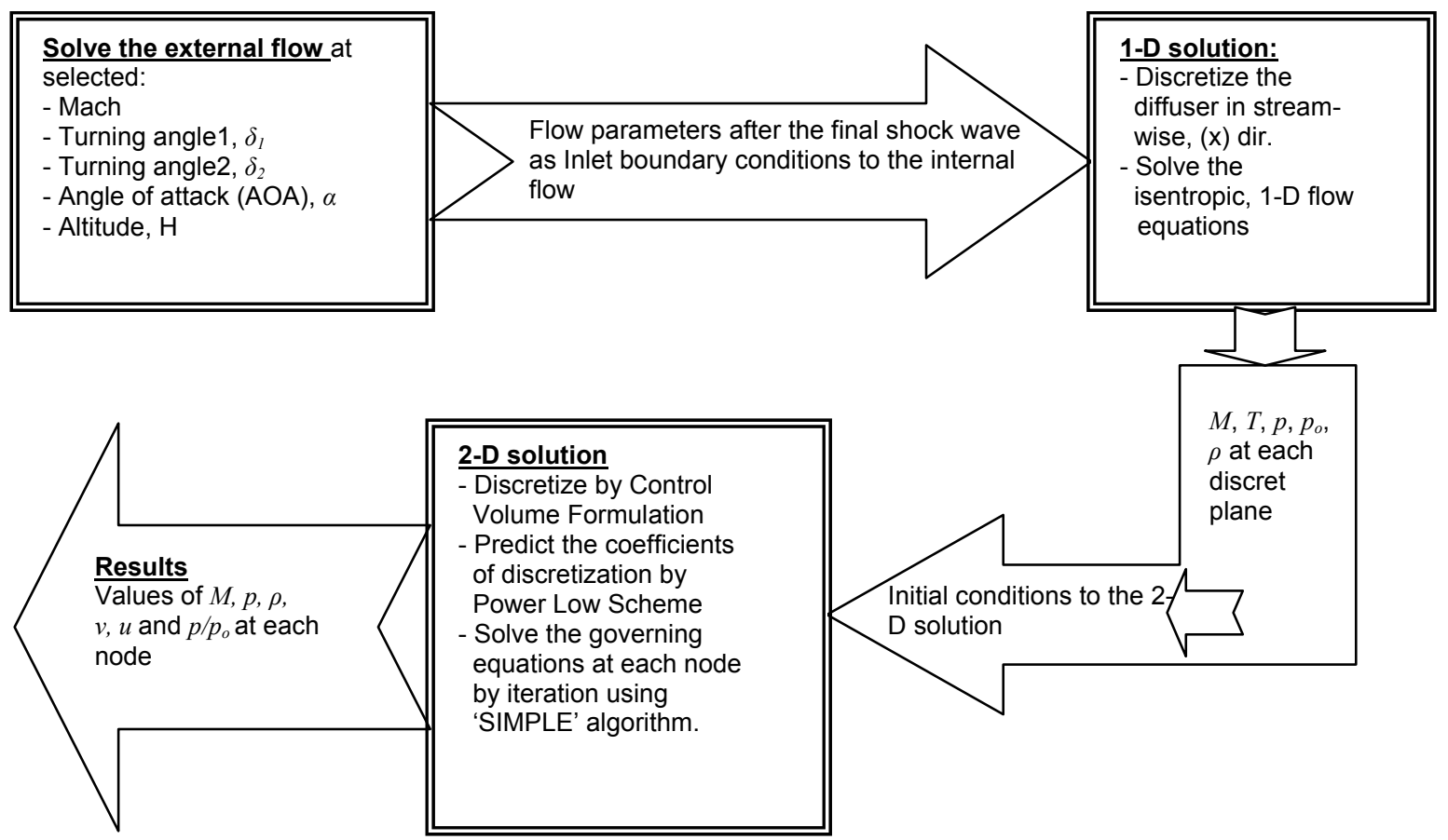

Fig.1. Methodology of the solution and analysis

\section{MODELING OF THE EXTERNAL FLOW}

The external flow is representing the regime of the supersonic flow subjected to changes once approaching the wedge of the spike. System of waves is created. It may consist of Oblique Shock Wave (OSW), Normal Shock Wave (NSW) and expansion wave, or so called Prandtl-Mayer Wave (P-MW). To solve this part of the flow, the followings are required to be modeled.

\section{Modeling of the Isentropic Flow}

The isentropic flow is the most assumed simple flow to simplify the solution of equations. It is very close to reality in engine intakes. It is characterized by frictionless and adiabatic flow. In this case, the local Mach number is the reference or characteristic parameter in compressible flow. Once the flow area is changed, the Mach value along the stream wise is changed and the flow properties are evaluated in ratio forms of the 
total to static values as: [1]

Pressure ratio, $p_{o} / p$; Density ratio, $\rho_{o} / \rho$; and Temperature ratio, $T_{o} / T=f(M)$

Where, $M$ is the local Mach No. The formulas are available in many literatures, e.g. [1], [16].

\section{Modeling of the Normal Shock Wave}

Usually, the up stream of the NSW is defined by $(x)$ and just down stream behind the wave by $(y)$. The equations appropriate to this flow are derived as function of the up stream Mach number $M x$. The stagnation temperature across the shock is constant.

Down stream Mach number:

$$
M_{y}=\sqrt{\frac{(\gamma-1) M_{x}{ }^{2}+2}{2 \gamma M_{x}{ }^{2}-(\gamma-1)}}
$$

Total pressure ratio:

$$
\frac{P o_{y}}{P o_{x}}=\left(\frac{2 \gamma}{\gamma+1} M_{x}^{2}-\frac{\gamma-1}{\gamma+1}\right)^{\frac{-1}{\gamma-1}}\left(\frac{(\gamma+1) M_{x}{ }^{2}}{2+(\gamma-1) M_{x}{ }^{2}}\right)^{\frac{\gamma}{\gamma-1}}
$$

The other flow properties are evaluated as

Pressure ratio, $p_{y} / p_{x}$; Density ratio, $\rho_{y} / \rho_{x}$; and Temperature ratio, $T_{y} / T_{x}=f\left(M_{x}\right)$

where, $M_{x}$ is the Mach No. of the flow just up stream of the NSW.

\section{Modeling of the Oblique Shock Wave}

The properties of flow behind the OSW are function of the up steam Mach number, say, $M_{1}$, and the shock angle $(\sigma)$ which could be found from the following relation:

$$
\tan \delta=\frac{2 \cot \sigma\left(M_{1}^{2} \sin ^{2} \sigma-1\right)}{2+M_{1}{ }^{2}(\delta+\cos 2 \sigma)}
$$

The equation cannot solve directly and iteration technique is the way to predict $\delta$. The down stream Mach number:

$$
M_{2}=\sqrt{\frac{(\gamma-1) M_{1}^{2} \sin ^{2} \sigma+2}{2 \gamma M_{1}{ }^{2} \sin ^{2} \sigma-(\gamma-1)} \frac{1}{\sin ^{2}(\sigma-\delta)}}
$$

Total pressure ratio: 
$\frac{P o_{2}}{P o_{1}}=\frac{P_{2}}{P_{1}}\left(\frac{1+\frac{\gamma-1}{2} M_{2}^{2}}{1+\frac{\gamma-1}{2} M_{1}^{2}}\right)^{\frac{\gamma}{\gamma-1}}$

Pressure ratio, $p_{2} / p_{1}$; Density ratio, $\rho_{2} / \rho_{1}$; and Temperature ratio, $T_{2} / T_{1}=f\left(M_{1}, \sigma\right)$

where, $M_{1}$ is the Mach No. upstream of the OSW and $\sigma$ is the shock angle.

\section{Modeling of Detached Shock Wave}

Detached shock wave occurs, when the flow pass a wedge with deflection angle, $\delta$ greater than maximum deflection angle for detail Mach number i.e. when $\delta>\delta_{\max } . \delta_{\max }$ can be found from the equation:

$$
\tan \delta_{\text {max }}=\frac{2 \cot \sigma_{\text {max }}\left(M_{1}^{2} \sin ^{2} \sigma_{\text {max }}-1\right)}{2+M_{1}^{2}\left(\delta_{\text {max }}+\cos 2 \sigma_{\text {max }}\right)}
$$

where, $\sigma_{\max }$ is the oblique wave angle obtained from:

$$
\sin ^{2} \sigma_{\max }=\frac{\gamma+1}{4 \gamma}-\frac{1}{\gamma M_{1}^{2}}\left[1-\sqrt{(\gamma+1)\left(1+\frac{\gamma-1}{2} M_{1}^{2}+\frac{\gamma+1}{16} M_{1}^{4}\right)}\right]
$$

The front side of the wedge is treated as normal shock wave. [16]

\section{Modeling of Prantdl- Mayer Waves}

If the angle of attack becomes higher than the wedge deflection angle the nature of flow is expanded. The change in the properties occurs gradually across a series of waves emanating at the surface.

The turning angle of the flow up stream to down stream of the P-MW is:

$\delta=v_{2}-v_{1}$

Where, $v_{1}=f\left(M_{1}\right)$ and $v_{2}=f\left(M_{2}\right)$ related as

$$
v=\sqrt{\frac{\gamma+1}{\gamma-1}} \tan ^{-1} \sqrt{\frac{\gamma-1}{\gamma+1}\left(M^{2}-1\right)-\tan ^{-1} \sqrt{M^{2}-1}}
$$

The change of properties across these waves is isentropic, so both the stagnation temperature and stagnation pressure are constants. Down stream Mach number $M_{2}$ can be found from equation 9 by iteration (substituting $v$ by $v_{2}$ ). The other properties can be found by using isentropic ratios at down stream Mach number. 


\section{MODELING OF THE INTERNAL FLOW}

Navier-Stokes equations under the assumptions of steady, viscous, compressible, and two-dimensional, without body forces or heat transfer can be derived in Cartesian coordinates as:

Continuity:

$$
\rho \frac{\partial u}{\partial x}+u \frac{\partial \rho}{\partial x}+\rho \frac{\partial v}{\partial y}+v \frac{\partial \rho}{\partial y}=0
$$

X- momentum:

$$
u \frac{\partial u}{\partial x}+v \frac{\partial u}{\partial y}+\frac{1}{\rho} \frac{\partial P}{\partial x}=\frac{\mu}{\rho}\left(\frac{\partial^{2} u}{\partial x^{2}}+\frac{\partial^{2} u}{\partial y^{2}}\right)
$$

Y- momentum:

$u \frac{\partial v}{\partial x}+v \frac{\partial v}{\partial y}+\frac{1}{\rho} \frac{\partial P}{\partial y}=\frac{\mu}{\rho}\left(\frac{\partial^{2} v}{\partial x^{2}}+\frac{\partial^{2} v}{\partial y^{2}}\right)$

Also, the state equation is required to link the density to the pressure. After derivation with respect to $x$ and $y$, the state equation becomes:

$$
\begin{aligned}
& \frac{\partial p}{\partial x}=R\left[\rho \frac{\partial T}{\partial x}+T \frac{\partial \rho}{\partial x}\right] \\
& \frac{\partial p}{\partial y}=R\left[\rho \frac{\partial T}{\partial y}+T \frac{\partial \rho}{\partial y}\right]
\end{aligned}
$$

The solution of these equations is complex, mainly, if the variables are required to be evaluated at large number of points in the flow field. The methods of the CFD technique are found to be the best tools to convert the PDE set of the governing equations to sets of algebraic equations which can be solved iteratively or by matrix inversions. In the present work, Patankar method [17], "SIMPLE algorithm", stands for "Semi-Empirical Method for Pressure-Linked Equation" is adopted, and, the domain is discretized by "CVF,. This method depends on discretization of the domain into a number of control volumes; each control volume contains main point (node) and neighbor points (nodes). The governing equations must be transformed from differential form to the discretization form and solved for each node. This requires two approximation schemes. The first approximation represents suitable guess of the flow variables at each node. The second approximation is used to evaluate the coefficients of the discretization equation.

\section{DISCRETIZATION AND GRID GENERATION}

One of the methods of deriving the discretization equation is the CVF. This method 
depends on discretization of the domain into a number of control volumes; each control volume contains main point (node $\mathrm{p}$ ) and neighbor points (nodes $\mathrm{nb}$ ).

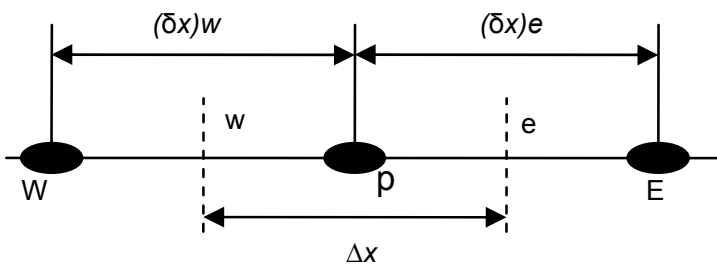

a. Grid point cluster for 1-D problem

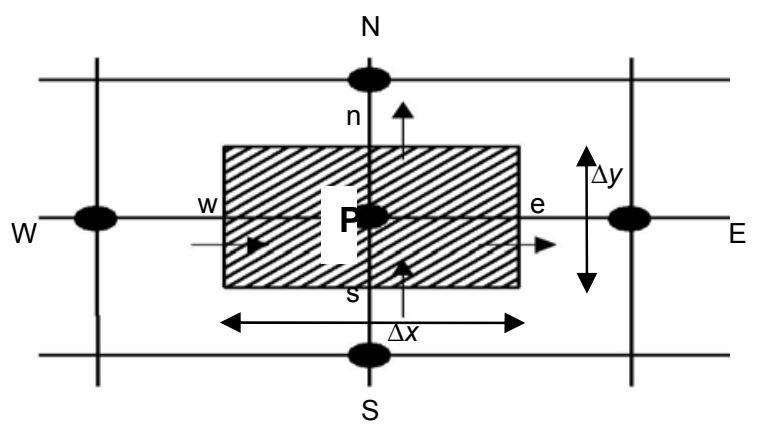

b. Control volume for 2-D problem

Fig.2. Nodal notation for the control volume grid generation

(E: east, W: west, N: north, S: south)

\section{Grid Generation}

For the 1-D solution, the domain is discretized in equally separated planes in the stream wise, $x$-direction. Each zy plane is parallel to the others and is perpendicular to the $\mathrm{x}$-direction of the flow. The domain is discretized to $\mathrm{n}$ subdivision in $\mathrm{x}$-direction with constant $\Delta x$. For the 2-D solution, each zy plane is discretized in y-direction and the properties are considered same in z-direction. The value of $\Delta y$ is variable from plane to another, but it is constant for all volumes located on same plane $(I)$, where:

$$
\Delta y(I)=\frac{y_{\max }(I)}{m}
$$

The resulting mesh from $n=20$, and, $m=20$ is shown in Fig.3.

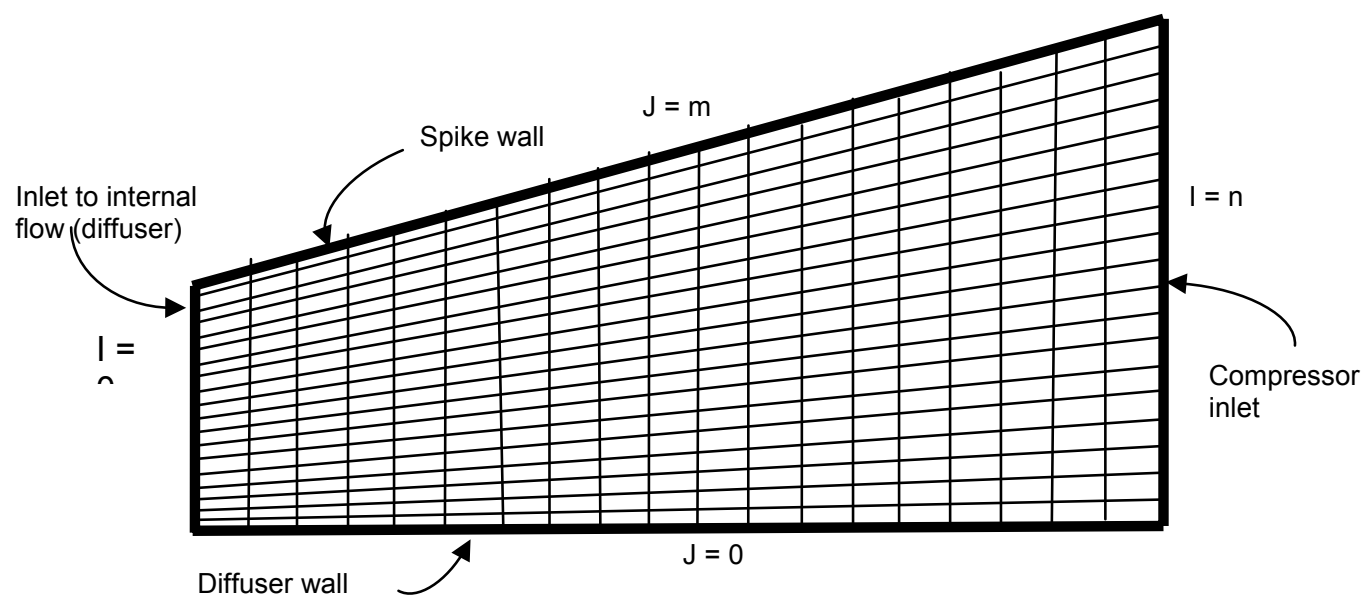

Fig.3. Grid generation for 2-D solution 


\section{Discretization of the Governing Equations}

For a variable $\Phi$ in the flow field, it is represented according to the CVF as:

$a_{P} \phi_{P}=a_{E} \phi_{E}+a_{W} \phi_{W}+a_{N} \phi_{N}+a_{S} \phi_{S}+b$

Where,

$$
a_{P}=a_{E}+a_{W}+a_{N}+a_{S}
$$

And

$$
a_{i}=\frac{\mu_{i} \Delta y}{(\delta x)_{i}}
$$

A better approximation to the exact solution is given by the power law scheme which is described for the present application by [18]. The power law expression, for $a_{E}$ for example, can be written as function of Peclet number, which is:

$$
P e_{e}=\frac{F_{e}}{D_{e}}
$$

where,

$$
F_{e}=(\rho u)_{e} \Delta y \quad \text { (17) } \quad \text { and } \quad D_{e}=\frac{\mu_{e} \Delta y}{(\delta x)_{e}}
$$

The values of $a_{E} / D_{e}=f\left(P e_{e}\right)$ are given by reference [17] for $\left(P e_{e}<-10\right),\left(-10<P e_{e}<\right.$ $0),\left(0<P e_{e}<10\right)$ and $\left(P e_{e}>10\right)$.

Accordingly, the momentum equations can be set in discretization form after manipulation and arrangement, as:

$u_{e}^{\prime}=\frac{A_{e}}{a_{e}}\left(P_{P}^{\prime}-P_{E}^{\prime}\right)$

and

$v_{n}^{\prime}=\frac{A_{n}}{a_{n}}\left(P_{P}^{\prime}-P_{N}^{\prime}\right)$

where

$$
\begin{gathered}
a_{e} u_{e}{ }^{*}=\sum a_{n b} u_{n b}{ }^{*}+b+\left(P_{P}-P_{E}\right) A_{e} \\
a_{n} v_{n}{ }^{*}=\sum a_{n b} v_{n b}{ }^{*}+b+\left(P_{P}-P_{N}\right) A_{n}
\end{gathered}
$$


And, the continuity equation, after integration over the control volume, becomes

$\left[(\rho u)_{e}-(\rho u)_{w}\right] \Delta y+\left[(\rho v)_{n}-(\rho v)_{s}\right] \Delta x=0$

If the velocity components are substituted, after rearrangement, the following discretization equation for ( $p$ ) would be obtained:

$a_{P} P_{P}^{\prime}=a_{E} P_{E}^{\prime}+a_{W} P_{W}^{\prime}+a_{N} P_{N}^{\prime}+a_{S} P_{S}^{\prime}+b^{\prime}$

$b^{\prime}=\left[\left(\rho u^{*}\right)_{w}-\left(\rho u^{*}\right)_{e}\right] \Delta y+\left[\left(\rho v^{*}\right)_{s}-\left(\rho v^{*}\right)_{n}\right] \Delta x$

Proposing that the corrected pressure, $p_{c}$ can be obtained from:

$p_{c}=p+p^{\prime}$

Then, the corresponding corrected velocities can be introduced in a similar manner, as:

$u=u^{*}+u^{\prime}$

$v=v^{*}+v^{\prime}$

It must hinted out that the corrected density should be related to the corrected pressure by using the state equation as,

$$
\rho=f(P)
$$

\section{COMPUTATIONAL PROCEDURE}

The computational procedure can be summarized in the following analysis steps.

\section{The free stream domain}

The analyses are carried out at operational altitude of $10000 \mathrm{~m}$. The corresponding properties of the free stream, the temperature $T_{\infty}$ and the pressure $\rho_{\infty}$, are evaluated from the aerodynamic relations [18] as:

$$
T_{\infty}=288.16-0.0065 H
$$

and

$$
P_{\infty}=101325\left(\frac{T_{\infty}}{188.16}\right)^{\left(\frac{0.03415 H}{188.16-T_{\infty}}\right)}
$$

\section{The external flow solution}

For each selected operational conditions (Mach, AOA and design forebody angle, the 
solution starts with the analysis of the external flow. The set of equations solving the external flow, analytically, have been converted to a computer program with many subroutines to solve all excepted wave types may present in the flow. The values of the flow variables, $M, P, P_{o}, T, V$, and $\rho$ obtained from the external flow solution are representing the inlet boundary conditions to the internal part of the inlet.

\section{1-D internal flow solution}

Subroutine DIM1 generates the nodal points. The internal subsonic flow domain is sub divided into $n=20$ equally spaced segments in the $x$-dir. Then, under the assumption of viscous, compressible, 1-D, the variables of the flow field are evaluated at each node using a set of derived equations ending with:

$$
\left.\begin{array}{l}
d M=M\left\{-\left[\frac{1+(\gamma-1) \frac{M^{2}}{2}}{1-M^{2}}\right] \frac{d A}{A}+\left[\frac{1+(\gamma-1) \frac{M^{2}}{2}}{1-M^{2}}\right] \frac{\gamma M^{2}}{2} \frac{4 f d x}{D_{H}}\right\} \\
D_{H}=\frac{4 \text { area }}{\text { Perimeter }}=\frac{4(y \times w)}{2(y+w)} \\
f=\frac{0.0625}{\left[\log \left(\frac{5.74}{\left.\operatorname{Re}^{0.9}\right)}\right]^{2}\right.} \\
M_{\text {out }}=M_{\text {in }}+d M \\
T_{\text {out }}=T_{\text {in }}+d T \\
V_{\text {out }}=V_{\text {in }}+d V \\
p_{\text {out }}=p_{\text {in }}+d P \\
\rho_{\text {out }}=\rho_{\text {in }}+d \rho
\end{array}\right]
$$

The 1-D solution provides preliminary values for the 2-D solution at each node in the domain.

\section{2-D internal flow solution}

The procedure for the calculation of the 2-D flow field is based on the "SIMPLE algorithm" described earlier.

The solution of the momentum equation will go to diverge unless some under-relaxation is used. In other words, instead of using equation (25a,b), employing:

$$
\begin{aligned}
& u=u^{*}+\alpha_{v} u^{\prime} \\
& v=v^{*}+\alpha_{v} v^{\prime}
\end{aligned}
$$

with $\alpha_{v}=0.5$, in the same manner, Eq. (24) replaced by: 
$p_{c}=p+\alpha_{p} p^{\prime}$

with $\alpha_{P}$ is set equal to 0.8

The sequence of operations is:

4.1 Generate the two-dimensional control volumes and the grid points.

4.2 Calculate the coefficients of discretization equation for each grid point using equations (13) to (18).

4.3 Solve equations $(19 a, b)$ and $(20 a, b)$, to find $u^{*}$ and $v{ }^{*}$ for all neighbor points.

4.4 Solve Eq. (22) and Eq. (23) to find $\mathrm{P}^{\prime}$ at each main grid point.

4.5 Calculate the corrected pressure from equation (34).

4.6 Calculate the corrected velocities from equations (32 and 33)

4.7 Go back to Eq. (19a,b) and find new $u^{\prime}, v^{\prime}$ at neighbors points.

4.8 Determine the corrected value of density using the state equation.

4.9 Treat the corrected values as new guessed values, return to step (4.3) and repeat the whole procedure until a converged solution is obtained.

The criterion to determine the convergence is $\zeta=0.22$.

$$
\zeta=\sqrt{(\Delta v)^{2}+(\Delta u)^{2}+(\Delta p)^{2}}
$$

where,

$$
\left.\begin{array}{l}
\Delta v=v(k)-v(k-1) \\
\Delta u=u(k)-u(k-1) \\
\Delta p=p(k)-p(k-1)
\end{array}\right]
$$

At each node, $\zeta$ is evaluated. When $\zeta$ reaches 0.22 , the program considers the solution of that point is stable. The final node $(19,19)$ would be the alarm node to terminate the iteration when its $\zeta$ reaches 0.22 .

\section{RESULTS AND DISCUSSION}

Analysis of various operational conditions at different AOA and the interference effects of the free stream Mach value and wedge angle is the subject of the present work. The total pressure recovery ratio, $\left(P_{\delta} / P_{O^{\infty}}\right)$ is the dominated parameter for comparison at different operational conditions. For confidence of understanding, Fig.4 shows the location of the windward side, leeside and cowl lip.

Discussion of the behavior of the total pressure recovery due to the changes of the AOA being more convenient by defining the effective deflection angle as:
$\delta_{e}=\delta-\alpha$
(33a) in the leeside
$\delta_{e}=\delta+\alpha$
in the windward side 
Before presenting the results, the numerical method adopted in the present analysis is verified by comparing with experimental analysis. The data taken from NACA Research Memorandum are the results of experimental investigation on conical spike supersonic intake at free stream Mach number from 1.5 to 2.2 and AOA from $0^{\circ}$ to $10^{\circ}$. The spikes at

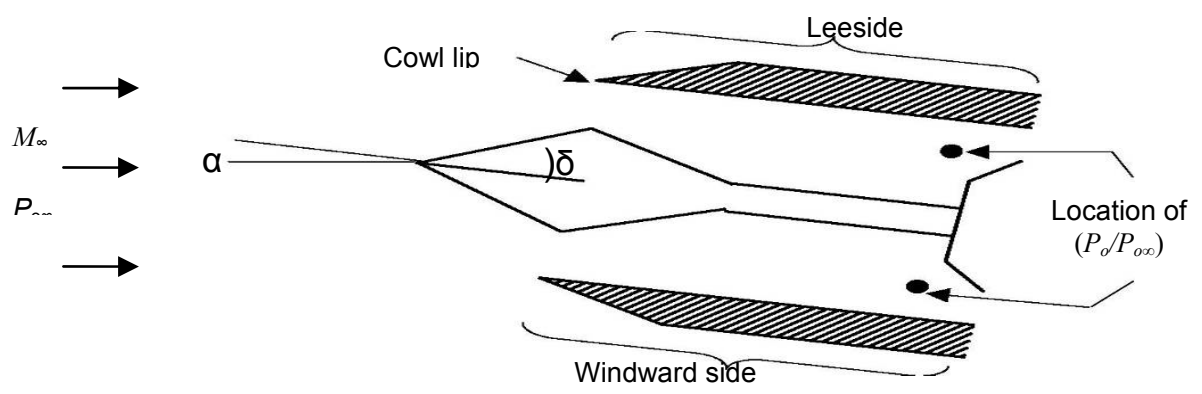

Fig.4. Outline of Supersonic Intakes

several deflection angles are investigated in the NACA Lewis 8-by-6 foot supersonic wind tunnel. Fig.5-a, indicates comparison at various Mach number at zero AOA. The trend of the present numerical and the experimental results of ref. [7] is the same. For Mach of 1.9, identical results are obtained for TPR. As Mach number increases, the numerical results of TPR are slightly higher than the experimental results.

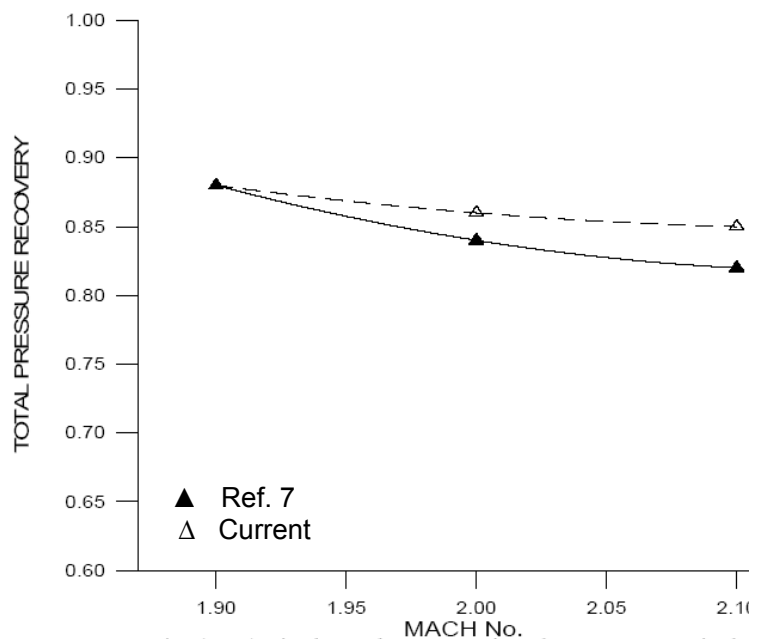

(a)

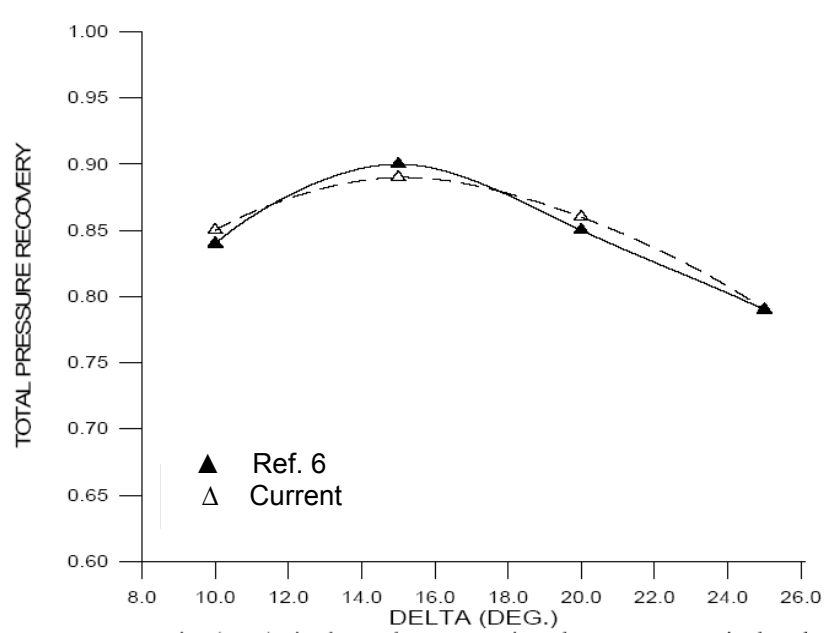

(b)

Fig.5. Comparison between present numerical results and previous experimental results of TPR, (a) At various Mach numbers, (b) At different forebody angles

As depicted in Fig.5-b, a very good agreement is claimed between the numerical and the experimental results of ref [6] within the entire tested spike forebody angles ranging from $10^{\circ}$ to $25^{\circ}$. The comparison is carried out at 2 Mach and zero AOA. Both analysis show same trend of increment in the TPR values until the deflection angle reaches its maximum value, then decreases. 
At positive effective deflection angle (greater than 0), the TPR in the leeside increases by increasing AOA as shown in Fig.6-a for Mach number (1.8) and Fig.6-b for Mach number (2.2). It means that there is a loosing in body compression, because the increase in AOA in the leeside reduces the effective deflection angle and that leads to reduce the Oblique shock angle becoming weaker shock. However, at negative effective deflection angle (less than 0 ) the total pressure recovery decreases by increasing AOA.

In the windward side, the TPR decreases by increasing angle of attack, because the increment in the effective deflection angle will lead to a stronger shock. See Fig.7-a for Mach number (1.8) and Fig.7-b for Mach number (2.2).

For free stream Mach number of 1.8, the change in the TPR becomes gradual, while in the high Mach number (2.2) the change in the TPR becomes precipitous.

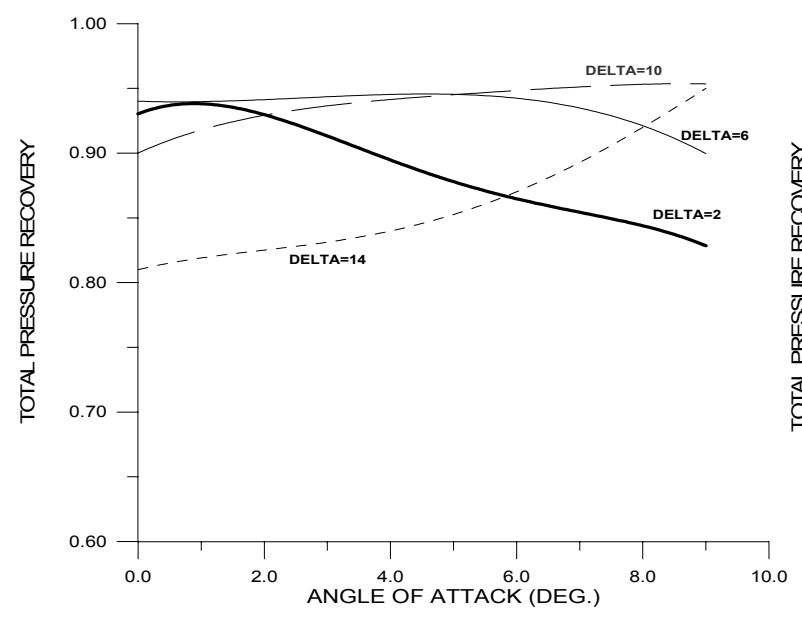

(a)

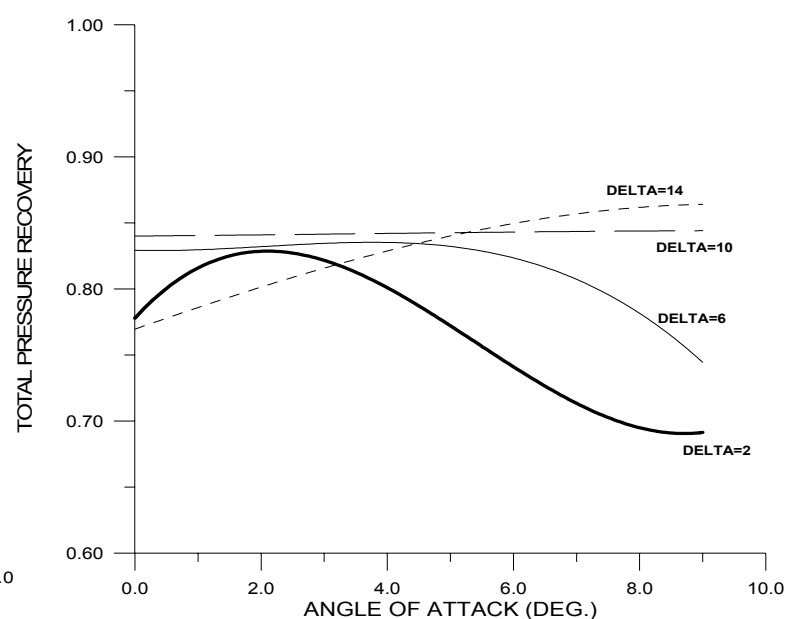

(b)

Fig.6. The effect of AOA on the TPR in the leeside, (a) at $M=1.8$, (b) at $M=2.2$.

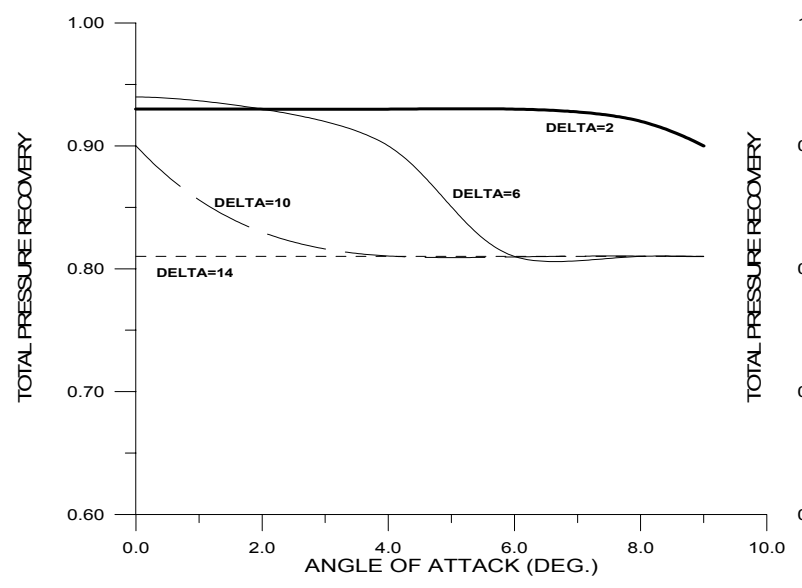

(a)

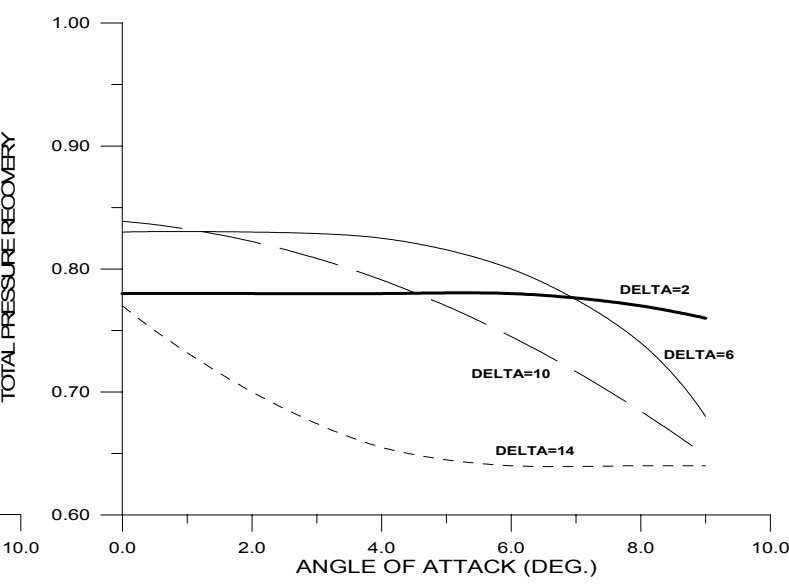

(b)

Fig.7. The effect of AOA on the TPR in the wind ward side, (a) at $M=1.8$, (b) at $M=2.2$. 
Generally speaking, to produce high TPR in the leeside, the effective deflection angle must be increased when the angle of attack increases. In the windward side, the inverse is the correct.

Results presented in Fig.8 represent a comparison between the experimental results from NACA, ref.[19], and the numerical results from the present analysis at Mach number (2.0) for $20^{\circ}$ wedge angle at leeside.

When the deflection angle increases the total pressure recovery increases also, because of increasing shock angle leads to increase the sequence of reflection shocks. This increasing is not continuous because of limitation of deflection angle, which leads to detached shock wave which stops the sequence of compression shocks.

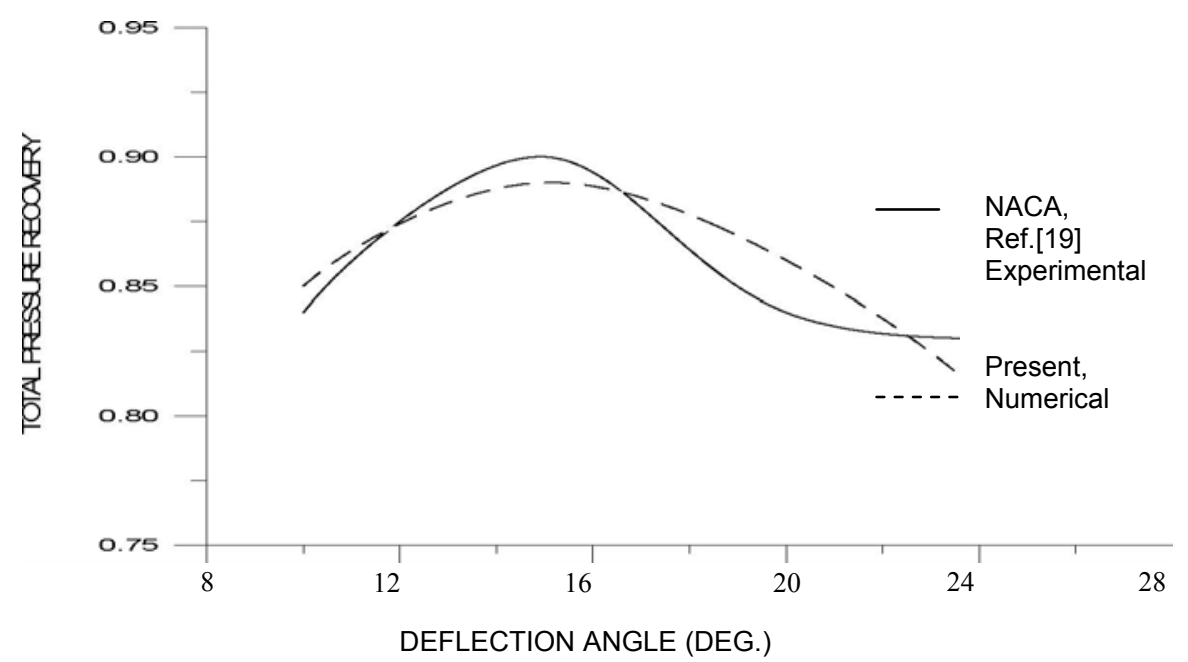

Fig.8. The effect of deflection angle on the total pressure recovery at the cowl lip in the leeside at $M=2$ and wedge angle $=20^{\circ}$

\section{CONCLUSIONS}

The flow through single deflected spiked supersonic intake is modeled by dividing the flow field into two regions. The external region which consists of the system of waves is modeled and analyzed analytically. The internal region which represents the expansion of the flow inside the diffuser is modeled and analyzed numerically. The "SIMPLE algorithm" is adopted for the grid generation and equations discretization under 2-D, viscous, compressible and steady flow assumption. The analysis has been proven to be able to characterize the supersonic intake and the TPR at the face of the compressor is predicted with good accuracy. The results show that the TPR increases at the windward side as the AOA increased, while it decreases in the leeside.

\section{ACKNOWLEDGEMENT}

Universiti Teknologi PETRONAS is acknowledged for supporting the publication of the present work financially and technically. 


\section{REFERENCES}

1. Patrick $\mathrm{H} \mathrm{O}$ and William E C "Compressible Fluid Flow", McGraw-Hill Company, (1997).

2. Air \& Space Magazine, Inventing the SR-71 Engine Inlets, Inlets|February, March, 1999 Supplem.htm.

3. Seddon, J and Gold Smith, E L "Intake Aerodynamics", AIAA Education Series, New York, (1985).

4. Englert, $\mathrm{G} W$ and Obery,J L "Evaluation of Five Conical Center-Body Supersonic Diffuser at Several Angels of Attack". NACA RM E51L04, (1952).

5. Gorton, G C, "Investigation of Translating-Spike Supersonic Inlet Means of MassFlow at Mach Number of 1.5, 1.8 and 2". NACA RM E53G10, (1953).

6. Jones, M L, Rebb L, and Simpkinson, S H "Drag Data for 16-Inch-Diameter Ramjet with Double-cone Inlet in Free Flight at Mach Number from 0.7 to 1.8', NACA RM E54H02, (1954).

7. Connor, J F and Meyer, R C "Performance Characteristics of Axisymmetric TwoCones and Isentropic Nose Inlets at Mach Number 1.9", NACA RM E55F29, (1955).

8. Biringen, $S$ "Numerical Simulation of Two-Dimensional Inlet flow Field", Journal of Aircraft, Vol.21,No.4, PP.244-249, (1984).

9. Moretti G "Efficient Euler Solver with Many Applications", AIAA Journal, Vol.26,No.6, PP.655-660, (1988).

10. Dudek, J C, Georgiadis, H J and Yoder, D A "Calculation of Turbulent Subsonic Diffuser Flows Using the NPARC Navier-Stokes Code", AIAA paper 96-0497, Jan., (1996).

11. Abbood, A H "Numerical Analysis of Two-Dimensional Convergence-Divergence Nozzle for A Modern Fighter", MSc. Thesis, College of Military Engineering, Baghdad, Iraq, (1999).

12. Yanta, W J, Collier A S, Spring C III, Boyd W, McArther J C "Experimental Measurements of the Flow in Scramjet Inlet at Mach 4", Journal of Propulsion, 6(6), pp. 784-790, (1990).

13. Gokhel, S S and Kumar, V R " Numerical Computation of Supersonic Inlet Flow", Int. J. Numer Meth Fluids, DOI: 10.1002fld.147, (2001).

14. Fayadh, M A "Numerical Prediction of Supersonic Inviscid and Viscous Flows over Arbitrary Configurations", PhD Thesis, University of Technology, Baghdad, Iraq, (2000).

15. Al-Kayiem H H and Sharief A S "Flow Analysis in Spiked Supersonic Intakes of A/C (I-Invicid)", Proceedings of AEROTECH-II, Conference on Aerospace Technology of XXI Century, Kuala Lumpur, Malaysia, 20-21 June (2007).

16. Michei A Saad "Compressible Fluid Flow", Prentice-Hall,INC, (1985).

17. Anderson D A, Tannehill, $\mathrm{R} \mathrm{H}$ and Plecher, $\mathrm{R} \mathrm{H}$ "Computational Fluid Mechanics and Heat Transfer", McGraw-Hill, (1984).

18. Salih, T W "The Flow Analysis of the Spiked Supersonic Intakes", M.Sc. Thesis, Mech. Eng. Dept., Al-Mustansiriya University, Baghdad, Iraq, (2002).

19. Connor, J F and Meyer, R C "Design Criteria for Axisymmetric and TwoDimensional Supersonic Inlets and Exits", NACA TN 3589, (1956). 